\title{
Advanced laboratory exercise: studying the dispersion properties of a prism pair
}

\section{T. Grósz, L. Gulyás, A. Kovács}

T. Grósz, L. Gulyás, A. P. Kovács, "Advanced laboratory exercise: studying the dispersion properties of a prism pair," Proc. SPIE 9793, Education and Training in Optics and Photonics: ETOP 2015, 97931N (8 October 2015); doi: $10.1117 / 12.2223123$

SPIE Event: Education and Training in Optics and Photonics: ETOP 2015, 2015, Bordeaux, France 


\title{
Advanced laboratory exercise: Studying the dispersion properties of a prism pair
}

\author{
T. Grósz*, L. Gulyás, A. P. Kovács \\ Department of Optics and Quantum Electronics, University of Szeged, \\ P.O. Box 406, Szeged, H-6701, Hungary
}

\begin{abstract}
A laboratory exercise has been developed in the frames of a new course called "Advanced undergraduate laboratory in femtosecond optics", which aims to study the dependence of the dispersion of a prism pair on the positions of the prisms. For the dispersion measurement we chose a relatively simple technique, called white light spectral interferometry. The prism pair consisting of two identical fused silica prisms was placed in the sample arm of a Michelson-interferometer illuminated with a tungsten halogen lamp. The interferograms were observed with a low resolution spectrometer in order to have a wide detection range (200-1100 nm). Measurements were performed by adjusting the optical path length in the second prism. The data was evaluated with the cosine-function fit method. Using the formalism of Fork the phase derivatives were theoretically calculated as well. The dependence of the dispersion coefficients on the displacement of the second prism agree well with the measurements. Using white light is advantageous as its broad wavelength range facilitates the retrieval of the spectral phase with high precision in a wide range. Furthermore, white light sources are relatively low-cost and safe in contrast to ultrashort laser sources.
\end{abstract}

Keywords: prism pair, white light spectral interferometry, chromatic dispersion

\section{INTRODUCTION}

Since the advent of lasers, constant effort is put toward producing ever shorter laser pulses, as they could facilitate the observation of processes on very short (femtosecond) time scale. The ultrashort pulse from the output of the laser is transmitted through or reflected from several optical elements until it reaches the target, and as a result it broadens in time which may affect the outcome of the experiment. There are different pulse-shaping devices at our disposal, such as pulse compressors, which provide a possibility of compressing the pulse to its original length. Theoretically every optical element that possesses angular dispersion can be used to compensate positive material dispersion. The most widespread pulse compressor devices consist of prism or grating pairs. ${ }^{1-4}$ Regarding the employment and features of the above mentioned compressors there are significant differences. Whereas prism compressors are mainly used to compensate the nonlinear phase shift caused by the material dispersion of the gain medium in a femtosecond laser oscillator, grating compressors are mainly used to compensate dispersion of greater magnitudes compared to what is achievable with prism compressors, for instance in CPA (Chirped Pulse Amplification) systems. Moreover, a notable distinction is that prism compressors have much lower loss than those consisting of gratings, provided that the apex angle of the prisms is chosen in such a way that the incident beam fulfills the Brewster-principle.

In ultrashort laser technology there is a great demand of controlling nonlinear dispersion terms, as certain pulse distortions can be associated with given terms, thus their retrieval is of tremendous advantage. Spectral interferometry is a widely used and simple procedure for dispersion characterization. One of its enticing features is that a halogen lamp can be used as broadband light source which is not that expensive and hazardous as an ultrashort laser would be. There are more evaluation techniques suitable for spectral phase retrieval, one of which is the cosine function fit (CFF) method. ${ }^{5,6}$

In this work we present an undergraduate laboratory exercise called "Dependence of the dispersion of a prism pair on the positions of the prisms" developed in the frames of a new course called "Advanced undergraduate laboratory in femtosecond optics" belonging to a specialization in Laser physics. The students have the opportunity to study the dispersion properties of a prism pair in different prism positions in a wide wavelength range and retrieve the coefficients

* tgrosz@ titan.physx.u-szeged.hu; phone +36 62 544-528; fax +36 62 544-658

Education and Training in Optics and Photonics: ETOP 2015, edited by Eric Cormier, Laurent Sarger Proc. of SPIE Vol. 9793, 97931N · C 2015 SPIE, IEEE, OSA, ICO · doi: 10.1117/12.2223123 
using the CFF method. Using the formalism of Fork et al. ${ }^{1}$ the phase derivatives are also calculated theoretically and they are compared with the results of the measurements.

\section{THEORY}

The basic idea of spectral interferometry is using a two-beam interferometer illuminated with a broadband light source, such as an ultrashort laser pulse or alternatively white light, for instance a halogen lamp. The optical element under study is placed in the sample arm of the interferometer, while the other arm serves as a reference with adjustable path length. At given $\tau$ time differences between the sample and reference arms interference fringes can be observed by a spectrometer placed at the output of the interferometer. The frequency-dependent intensity distribution $I(\omega)$ of the interference pattern is the following:

$$
I(\omega)=I_{r}(\omega)+I_{s}(\omega)+2 \sqrt{I_{r}(\omega) \cdot I_{s}(\omega)} \cdot \cos (\Phi(\omega)),
$$

where $I_{r}$ and $I_{s}$ are the spectral intensities of the reference and the sample arms, respectively, and $\Phi$ denotes the spectral phase difference between the arms:

$$
\Phi(\omega)=\varphi(\omega)+\omega \tau
$$

During this laboratory exercise the dispersion properties of a prism pair are studied. In order to do so, first we need to recall which properties can be used to describe the linear propagation of an ultrashort pulse in a homogeneous, isotropic, dispersive medium. Conventionally, as in the literature the dispersion of an optical element is characterized by the coefficients in the Taylor-series of its spectral phase $\varphi(\omega)$ around the carrier frequency $\omega_{0}$ :

$$
\varphi(\omega) \approx \varphi\left(\omega_{0}\right)+\left.\sum_{n=1}^{N} \frac{1}{n !} \frac{d^{n} \varphi}{d \omega^{n}}\right|_{\omega_{0}}\left(\omega-\omega_{0}\right)^{n},
$$

where

$$
\begin{gathered}
G D\left(\omega_{0}\right)=\left.\frac{d \varphi}{d \omega}\right|_{\omega=\omega_{0}}, \operatorname{GDD}\left(\omega_{0}\right)=\left.\frac{d^{2} \varphi}{d \omega^{2}}\right|_{\omega=\omega_{0}}, \\
\operatorname{TOD}\left(\omega_{0}\right)=\left.\frac{d^{3} \varphi}{d \omega^{3}}\right|_{\omega=\omega_{0}}, \operatorname{FOD}\left(\omega_{0}\right)=\left.\frac{d^{4} \varphi}{d \omega^{4}}\right|_{\omega=\omega_{0}}
\end{gathered}
$$

is the group-delay (GD), the group-delay dispersion (GDD), the third-order dispersion (TOD) and the fourth-order dispersion (FOD), respectively.

There are more possibilities for spectral phase retrieval one of which is the CFF method. The first step of the evaluation is to perform the following normalization:

$$
\cos (\Phi(\omega))=\frac{I(\omega)-I_{r}(\omega)-I_{s}(\omega)}{2 \sqrt{I_{r}(\omega) \cdot I_{s}(\omega)}}
$$

When this normalized spectral interferogram is obtained, it can be fitted with an appropriate order of phase-modulated cosine function: 


$$
I_{f i t}=c_{1}+c_{2} \cos \left(b_{0}+b_{1} \Delta \omega+b_{2} \Delta \omega^{2}+b_{3} \Delta \omega^{3}+b_{4} \Delta \omega^{4}\right)
$$

where $b_{0}, b_{1} \ldots$ correspond to the coefficients in the Taylor-series in the following way: $b_{1}=\mathrm{GD}, b_{2}=\mathrm{GDD} / 2, b_{3}=$ $\mathrm{TOD} / 6$ and $b_{4}=\mathrm{FOD} / 24 . c_{1}$ and $c_{2}$ are fitting parameters.

As an ultrashort pulse travels through an optical element exhibiting angular dispersion, for example through a prism, the spectral components belonging to different frequencies travel along different directions after the element. This means, that there is a phase difference between the components even though the monochromatic waves travel with the same speed, but in different directions. In this case the measured GDD at a given part of the pulse arises partly from the propagation in glass, and partly from angular dispersion. The pulse broadens in time while propagating in the glass, as in visible light the majority of materials have positive GDD. Angular dispersion on the other hand results in negative GDD. If we were to use only one prism, the pulse would be compressed in time, however, as the GDD caused by angular dispersion is proportional to the distance from the prism, the pulse would be the shortest only at a given point. A further problem is that in this case the beam diverges and a so-called spatial chirp occurs, thus the spectrum of the pulse changes greatly in its cross-section. For these reasons prism pairs are used as pulse compressors (Fig. 1). The prisms should be entirely identical, isosceles, made of the same material and have equal apex angles. The prisms should be placed in opposing directions, in such a way that, to any face of one prism a corresponding parallel face of the other prism can be found. Doing so the second prism compensates the angular dispersion caused by the first one, thus after leaving the second prism all spectral components are parallel as it can be seen in Fig. 1(a). To combine these parallel but spatially separated components into one pulse once again, an additional prism pair is necessary (Fig. 1(b)). An equivalent, but a much more cost-effective realization is to use two prisms and place a plane mirror after the second one, thus sending the beam twice through the system, as it can be seen in Fig. 1(a).

a)
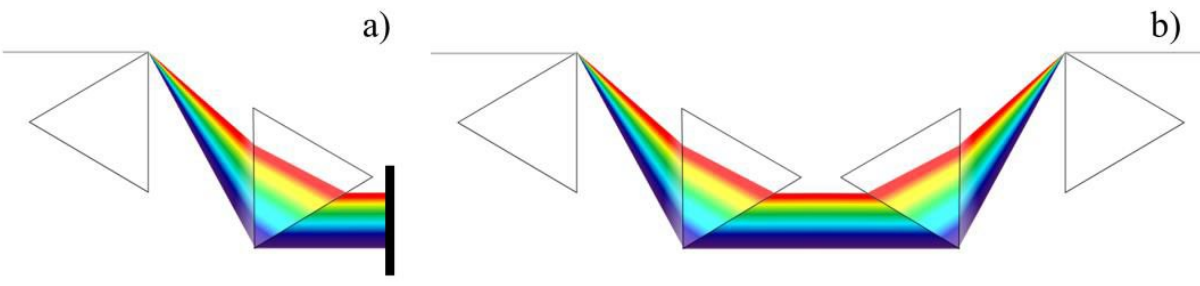

Figure 1. Prism pair with (a) two and with (b) four prisms.

The GDD of such systems is determined by several factors, partly by the propagation in the prisms, and additionally by the change in the optical path-length in each prism and between the two prisms due to angular dispersion. As it can be seen, it is quite complicated to calculate the optical path lengths and the phase shift in such systems, thus the retrieval of the phase derivatives is also problematic.

In order to determine the dispersion coefficients of a prism pair there is a far easier solution. As a means to understand this, the work of Fork et al. will be reviewed. ${ }^{1}$ First of all, we calculate the phase shift of the prism pair in Fig. 2 for a whole round trip:

$$
\varphi_{p p}(\omega)=2 \frac{\omega}{c} L_{0} \cos (\beta)
$$

where $c$ is the speed of light in vacuum, $L_{0}$ is the distance between the two prisms and $\beta$ is the angle between the propagating beam and the linear connecting the two apices of the prisms. 


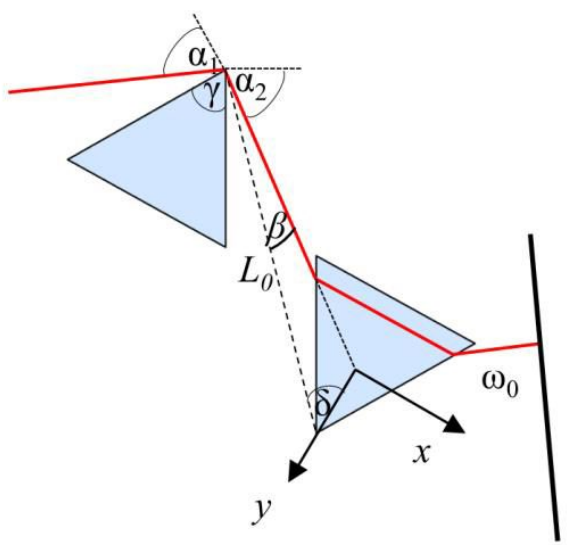

Figure 2. Prism pair with a plane mirror indicating the angles and the coordinate-system used in the calculations.

By the derivation of Eq. (7) with respect to angular frequency the GD, and by a further derivation the GDD is obtained:

$$
\begin{gathered}
G D_{p p}(\omega)=2 \frac{L_{0}}{c}\left[\cos (\beta)-\omega \sin (\beta) \frac{d \beta}{d \omega}\right], \\
G D D_{p p}(\omega)=-2 \frac{L_{0}}{c}\left[\left(2 \frac{d \beta}{d \omega}+\omega \frac{d^{2} \beta}{d \omega^{2}}\right) \sin (\beta)+\omega\left(\frac{d \beta}{d \omega}\right)^{2} \cos (\beta)\right] .
\end{gathered}
$$

If $\beta\left(\omega_{0}\right)=0$, i.e. the component belonging to the carrier frequency $\omega_{0}$ propagates exactly at the apices of the two prisms, the GDD at $\omega_{0}$ is the following:

$$
G D D_{p p}\left(\omega_{0}\right)=-2 \frac{L_{0} \omega_{0}}{c}\left(\left.\frac{d \beta}{d \omega}\right|_{\omega_{0}}\right)^{2}
$$

In this case, as it can be seen from Eq. (10), the GDD of a prism pair is negative and it is only determined by the distance between the two prisms and the angular dispersion of the prisms. Provided, that the beam in the second prism travels near the apex, i.e. $\beta$ is small, an approximation can be deduced for the GDD according to Eq. (9):

$$
\left.G D D_{p p}\left(\omega_{0}\right) \approx-2 \frac{L_{0}}{c} \mid\left(\left.2 \frac{d \beta}{d \omega}\right|_{\omega_{0}}+\left.\omega \frac{d^{2} \beta}{d \omega^{2}}\right|_{\omega_{0}}\right) \beta\left(\omega_{0}\right)+\omega_{0}\left(\left.\frac{d \beta}{d \omega}\right|_{\omega_{0}}\right)^{2}\right]
$$

Based on Fig. 2 and applying the sine rule we get

$$
\frac{y}{L_{0}}=\frac{\sin (\beta)}{\sin (\delta)} \approx \frac{\beta}{\cos \left(\alpha_{2}-\frac{\gamma}{2}+\beta\right)} \approx \frac{\beta}{\cos \left(\alpha_{2}-\frac{\gamma}{2}\right)},
$$

from which follows 


$$
\beta \approx \frac{\cos \left(\alpha_{2}-\frac{\gamma}{2}\right)}{L_{0}} y .
$$

Substituting Eq. (13) into Eq. (11) the GDD can be written in the form

$$
G D D_{p p}\left(\omega_{0}\right) \approx m y+b,
$$

where

$$
\begin{gathered}
m=-2 \frac{\cos \left(\alpha_{2}\left(\omega_{0}\right)-\frac{\gamma}{2}\right)}{c}\left(\left.2 \frac{d \beta}{d \omega}\right|_{\omega_{0}}+\left.\omega_{0} \frac{d^{2} \beta}{d \omega^{2}}\right|_{\omega_{0}}\right) \\
b=-2 \frac{L_{0} \omega_{0}}{c}\left(\left.\frac{d \beta}{d \omega}\right|_{\omega_{0}}\right)^{2} .
\end{gathered}
$$

Using a similar scheme, the TOD can also be written in a form

$$
T O D_{p p}\left(\omega_{0}\right) \approx m y+b,
$$

where

$$
\begin{gathered}
m=-2 \frac{\cos \left(\alpha_{2}\left(\omega_{0}\right)-\frac{\gamma}{2}\right)}{c}\left(\left.\omega_{0} \frac{d^{3} \beta}{d \omega^{3}}\right|_{\omega_{0}}+\left.3 \frac{d^{2} \beta}{d \omega^{2}}\right|_{\omega_{0}}-\omega_{0}\left(\left.\frac{d \beta}{d \omega}\right|_{\omega_{0}}\right)^{3}\right) \\
b=-2 \frac{L_{0}}{c}\left(\left.\left.3 \omega_{0} \frac{d \beta}{d \omega}\right|_{\omega_{0}} \frac{d^{2} \beta}{d \omega^{2}}\right|_{\omega_{0}}+3\left(\left.\frac{d \beta}{d \omega}\right|_{\omega_{0}}\right)^{2}\right) .
\end{gathered}
$$

The FOD of the prism pair takes the following form:

$$
F O D_{p p}\left(\omega_{0}\right) \approx m y+b,
$$

where

$$
m=-2 \frac{\cos \left(\alpha_{2}\left(\omega_{0}\right)-\frac{\gamma}{2}\right)}{c}\left(\left.\omega_{0} \frac{d^{4} \beta}{d \omega^{4}}\right|_{\omega_{0}}+\left.4 \frac{d^{3} \beta}{d \omega^{3}}\right|_{\omega_{0}}-\left.6 \omega_{0}\left(\left.\frac{d \beta}{d \omega}\right|_{\omega_{0}}\right)^{2} \frac{d^{2} \beta}{d \omega^{2}}\right|_{\omega_{0}}-4\left(\left.\frac{d \beta}{d \omega}\right|_{\omega_{0}}\right)^{3}\right)
$$




$$
b=-2 \frac{L_{0}}{c}\left(\left.\left.4 \omega_{0} \frac{d \beta}{d \omega}\right|_{\omega_{0}} \frac{d^{3} \beta}{d \omega^{3}}\right|_{\omega_{0}}+\left.\left.12 \frac{d \beta}{d \omega}\right|_{\omega_{0}} \frac{d^{2} \beta}{d \omega^{2}}\right|_{\omega_{0}}+3 \omega_{0}\left(\left.\frac{d^{2} \beta}{d \omega^{2}}\right|_{\omega_{0}}\right)^{2}\right)
$$

As it can be seen, the GDD, the TOD and the FOD of the system can be easily tuned. One possibility is to change the distance $L_{0}$ between the prisms (see Eq. (15), (17) and (19)), as the absolute value of each dispersion coefficient increases with growing distance. Another approach is to shift the second prism in parallel with the bisector of its apex, i.e. to change the value of $y$. From Eq. (14), (16) and (18) it is apparent that the GDD, the TOD and the FOD are all linearly related to $y$, as it is observable in Fig. 3, too. Furthermore, $\beta$ also increases with $y$. Assuming, that in the beginning the beam travels very close to the apex of the second prism, hence $\beta$ is small, the first term in Eq. (11) drops out. In the decisive second term $d \beta / d \omega$ is negative, its square is positive, and as it is multiplied by a negative number, the GDD will be negative. As $y$ and $\beta$ increases the importance of the first term becomes more and more pronounced. Here both terms are negative but as they are multiplied by a negative number, the term is overall positive, and slowly exceeds the absolute value of the second term, resulting in a positive GDD. This is in accordance with the conception that increasing optical path length in the second prism produces increasing positive GDD. In the case of the TOD and higher-orders it is harder to determine how the sign changes with increasing $\beta$, however, modeling can shad a light on such issues for a specific material. Fig. 3 shows the results of a simulation performed for a fused silica prism pair, with apex angles of $67.5^{\circ}$, placed $40 \mathrm{~cm}$ apart and fulfilling the minima deviation condition at $800 \mathrm{~nm}$. As it can be seen, in the given conditions as $y$ and $\beta$ are increased the TOD, just like the GDD, switches from negative to positive and FOD stays negative all along.

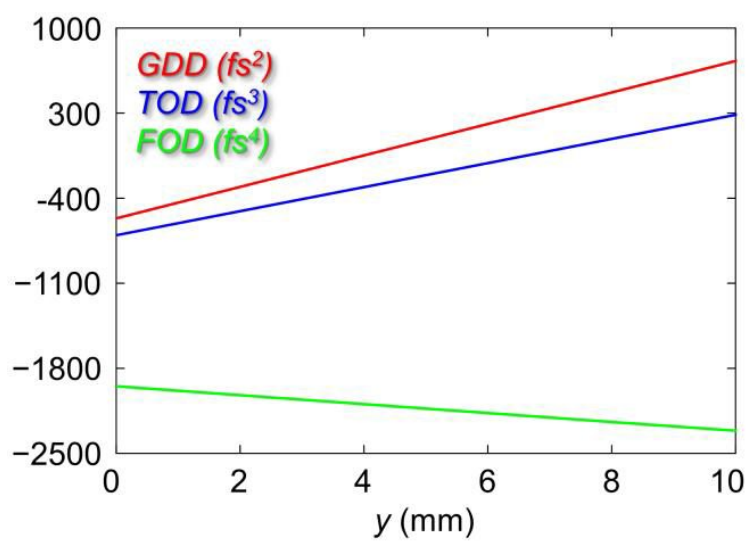

Figure 3. The dependence of the dispersion coefficients on the position of the second prism. The path length in the second prism is minimized at $0 \mathrm{~mm}$ and maximized at $10 \mathrm{~mm}$.

As can be concluded from the above, if $L_{0}$ and $\beta$ are known the phase shift can be calculated according to Eq. (7) and the dispersion coefficients can be retrieved by its numerical differentiation. Since neither the path of the beam in the second prism nor $\beta$ can be exactly determined, it is difficult to compare the results of the numerical differentiation with the measurements. However, as the translation of the second prism can be precisely measured, the slope of the dispersion curves can be determined according to Eq. (15), (17) and (19) and compared with those from the measurements. The calculations can be simplified by considering, that

$$
\frac{d \beta}{d \omega}=-\frac{d \alpha_{2}}{d \omega}
$$




\section{EXPERIMENTAL SETUP}

The dispersion of the prism pair is studied using spectral interferometry employing a Michelson-interferometer and a spectrometer (Fig. 4).

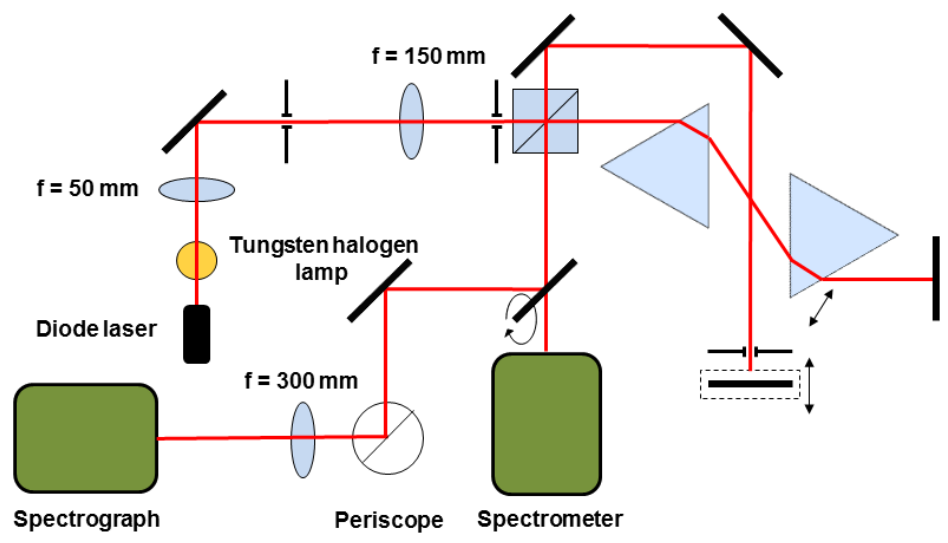

Figure 4. Experimental setup.

Instead of a femtosecond laser a common tungsten halogen lamp, thus white light illumination is used. The detailed tool list is presented in Table 1. During the measurement interferograms, as well as reference and sample spectra are recorded at different prism positions. The evaluation is performed with the CFF method.

Table 1. Tools required for the measurement.

\section{TOOLS}

Diode laser (2 mW)

Tungsten halogen lamp (100 W)

Converging lenses of 50, 150 and $300 \mathrm{~mm}$ focal length

(1-1 pcs.)

Beam splitter cube

Plane mirrors

$(8+1$ pcs. flip mirror $)$

Fused silica prisms of $67.5^{\circ}$ apex angle

(2 pcs.)

Irises

Linear translation stage

(3 pcs.)

Opto-mechanic components

Avantes 3648 spectrometer with the AvaSoft 7.6 for USB2 program

Imaging spectrograph (CEOptics)

Cosfit.xmcd program 


\section{RESULTS}

\subsection{Task 1}

The students need to assemble the experimental setup seen in Fig. 4 using the diode laser. First the prisms need to be placed $40 \mathrm{~cm}$ apart from each other, and the beam should pass near the apices of each prism. In the beginning the linear translator of the second prism should be set to $0 \mathrm{~mm}$. After the setup is complete the laser should be replaced with the halogen lamp. Before the measurements the residual angular dispersion should be cancelled with the help of an imaging spectrograph.

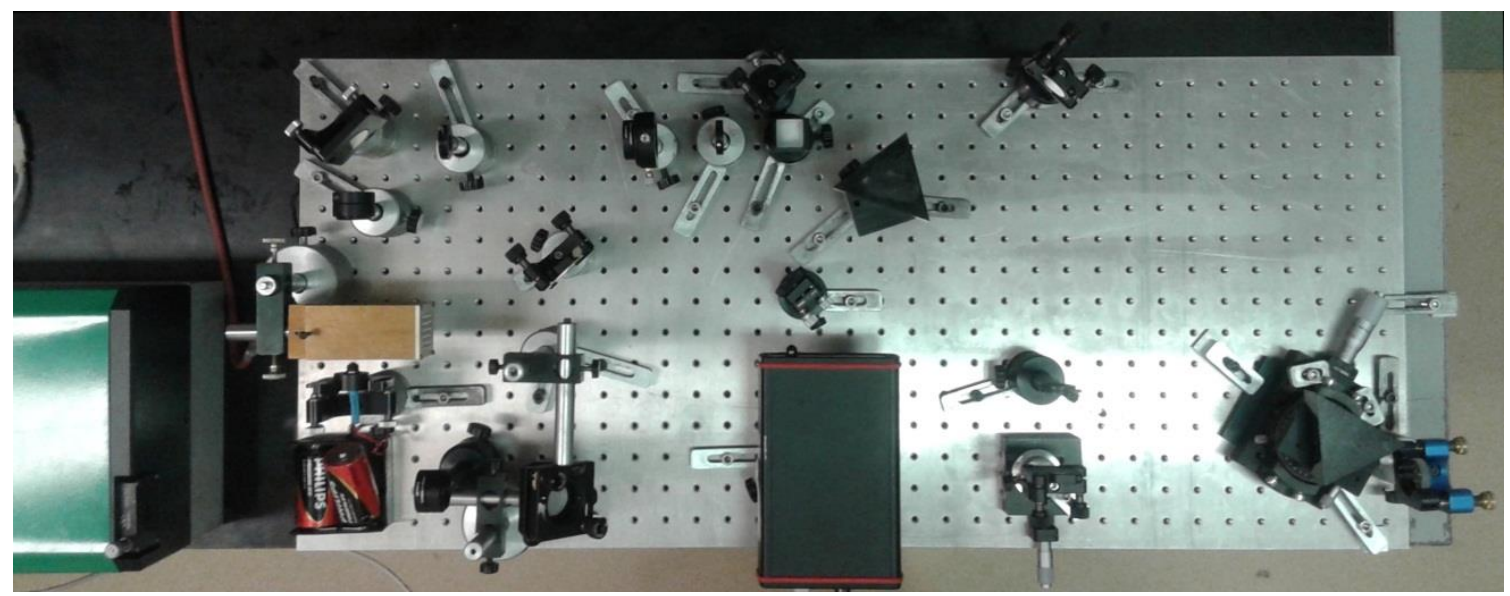

Figure 5. The realized experimental setup.

\subsection{Task 2}

In the next step the dispersion of the prism pair is measured in different positions of the second prism by recording interferograms, reference and sample spectra at various time delays using the spectrometer and employing white light illumination. Note that the setup was assembled with the diode laser, thus it is well set for a beam having a wavelength around $655 \mathrm{~nm}$, meaning that at $0 \mathrm{~mm}$ the red part of the spectrum travels at the apices of the prisms. As it can be seen, for practical reasons the measurement with white light should begin after the second prism was pushed a bit further into the beam path in order to avoid chopping off the spectrum. Accordingly, the measurements were conducted at $y=2,4,6$, 8 and $10 \mathrm{~mm}$.

\subsection{Task 3}

The recorded interferograms are evaluated with the CFF method using MathCad. Fig. 6 shows an example of a recorded and normalized interferogram with the cosine function fit. The dispersion coefficients are determined at $800 \mathrm{~nm}$ up to the fourth order. Results are shown in Table 2 below. As the second prism is pushed further into the beam, i.e. more glass is introduced to the system, the GDD slowly changes its sign from negative to positive. At some point the sign of the TOD also changes to positive, but the FOD stays negative the whole time. The coefficients are plotted against the position of the second prism (Fig. 7) and after a linear fit the slopes are determined (Table 3).

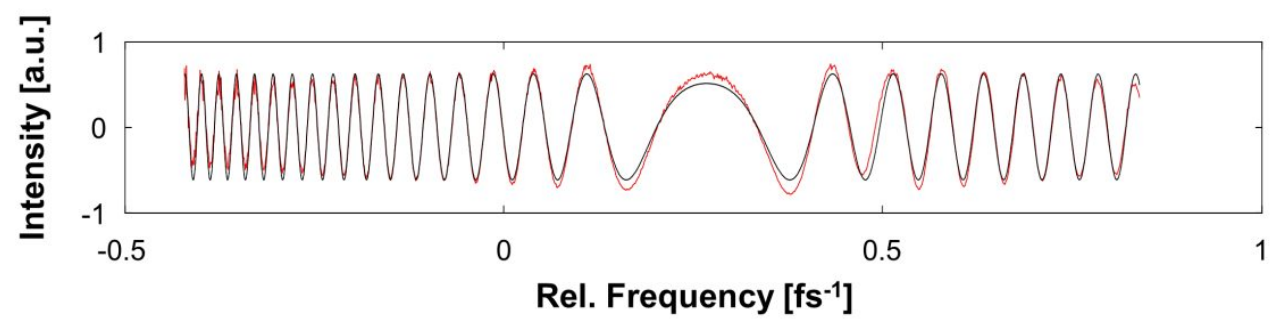

Figure 6. A recorded and normalized interferogram (red) with the cosine function fit (black). 
Table 2. Dispersion coefficients of a prism pair in different position of the second prism.

\begin{tabular}{|c|c|c|c|}
\hline$y[\mathrm{~mm}]$ & GDD $\left[\mathrm{fs}^{2}\right]$ & TOD $\left[\mathrm{fs}^{3}\right]$ & FOD $\left[\mathrm{fs}^{4}\right]$ \\
\hline 2 & $-259 \pm 2$ & $-475 \pm 8$ & $-2019 \pm 116$ \\
\hline 4 & $-12 \pm 1$ & $-249 \pm 5$ & $-1884 \pm 46$ \\
\hline 6 & $230 \pm 1$ & $-73 \pm 8$ & $-1857 \pm 28$ \\
\hline 8 & $472 \pm 1$ & $132 \pm 4$ & $-1976 \pm 61$ \\
\hline 10 & $714 \pm 1$ & $310 \pm 4$ & $-1984 \pm 46$ \\
\hline
\end{tabular}

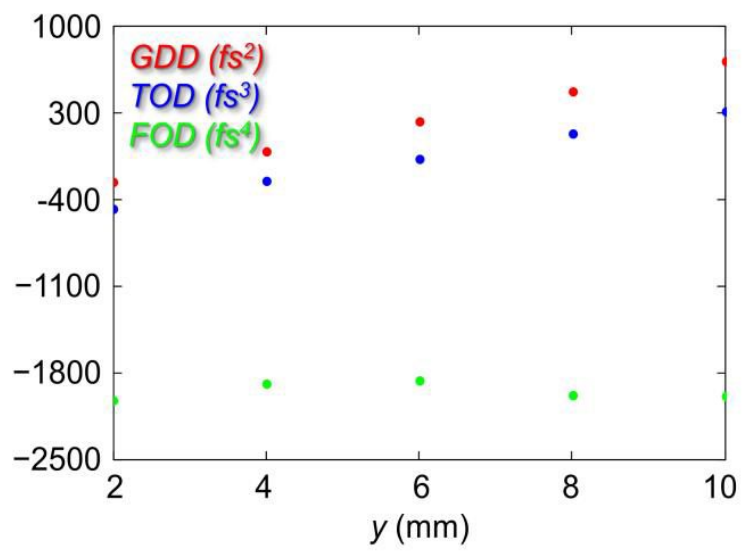

Figure 7. The measured dependence of the dispersion coefficients on the position of the second prism. The path length in the second prism is minimized at $0 \mathrm{~mm}$ and maximized at $10 \mathrm{~mm}$.

\subsection{Task 4}

After the necessary geometrical parameters are measured, the dependence of the dispersion coefficients on the position of the second prism can be determined using a MathCad calculation. The calculated slopes of the dispersion curves are compared with the measured slopes. As it can be seen from Table 3 the determined slopes agree well in the case of the TOD, differ a bit for the GDD, and greatly in the case of the FOD. As it is known, the coefficients of even (and also the odd) orders in the spectral phase are correlated to one another thus the large difference in the slope of the FOD can be interpreted as a result of the variance seen in the slope of the GDD. Additionally, the FOD was retrieved with the biggest error which resulted in a faulty slope.

Table 3. Measured and calculated slopes of the dispersion coefficients.

\begin{tabular}{|c|c|c|c|}
\hline & $\begin{array}{c}\text { Slope of the GDD } \\
{\left[\mathrm{fs}^{2} / \mathrm{mm}\right]}\end{array}$ & $\begin{array}{c}\text { Slope of the TOD } \\
{\left[\mathrm{fs}^{3} / \mathrm{mm}\right]}\end{array}$ & $\begin{array}{c}\text { Slope of the FOD } \\
{\left[\mathrm{fs}^{4} / \mathrm{mm}\right]}\end{array}$ \\
\hline Measurement & 121.5 & 98 & -1 \\
\hline Calculation & 129.4 & 99 & -36.7 \\
\hline
\end{tabular}

\section{SUMMARY}

In this work a detailed investigation of dispersion properties of a prism pair is presented. During the laboratory exercise the students become acquainted with white light spectral interferometry and the cosine-function fit, a common spectral phase retrieval method. The results of the measurements are compared with the theoretically calculated phase derivatives using MathCad. The difference between the values of the slopes in the case of the GDD and the FOD can be described by the overall correlation of the even coefficients, thus if one of them is retrieved with error it affects the other. 


\section{ACKNOWLEDGEMENTS}

The project was partially funded by TÁMOP-4.1.1.C-12/1/KONV-2012-0005 - „Preparation of the concerned sectors for educational and R\&D activities related to the Hungarian ELI project." which is supported by the European Union and cofinanced by the European Social Fund.

\section{REFERENCES}

[1] Fork R. L., Martinez O. E. and Gordon J. P., "Negative dispersion using pairs of prisms," Opt. Lett. 9, 150-152 (1984).

[2] Fork R. L., Brito Cruz C. H., Becker P. C., and Shank C. V., "Compression of optical pulses to six femtoseconds by using cubic phase compensation," Opt. Lett. 12, 483-485 (1987).

[3] Diels J.-C. and Rudolph W., [Ultrashort Lase Pulse Phenomena], Elsevier Inc., USA, 94-117 (2nd edition, 2006).

[4] Bor Zs. and Rácz B., "Group Velocity Dispersion in Prisms and its Application to Pulse Compression and Travelling-wave Excitation," Opt. Commun. 54, 165-170 (1985).

[5] Ye, Q., Xu, Ch., Liu, X., Knox, W. H., Yan, M. F., Windeler, R. S. and Eggleton, B., "Dispersion measurement of tapered airsilica microstructure fiber by white-light interferometry," Appl. Opt. 41(22), 4467-4470 (2002).

[6] Kardas, T. M. and Radzewicz, C., "Broadband near-infrared fibers dispersion measurement using white light interferometry," Opt. Commun. 282, 4361-4365 (2009). 